MATHEMATICS OF COMPUTATION

Volume 75, Number 256, October 2006, Pages 1721-1733

S 0025-5718(06)01863-1

Article electronically published on June 19, 2006

\title{
A POSSIBLE COUNTEREXAMPLE TO WELL POSEDNESS OF ENTROPY SOLUTIONS AND TO GODUNOV SCHEME CONVERGENCE
}

\author{
VOLKER ELLING
}

\begin{abstract}
A particular case of initial data for the two-dimensional Euler equations is studied numerically. The results show that the Godunov method does not always converge to the physical solution, at least not on feasible grids. Moreover, they suggest that entropy solutions (in the weak entropy inequality sense) are not well posed.
\end{abstract}

\section{INTRODUCTION}

Consider the Cauchy problem for a system of hyperbolic conservation laws,

$$
\begin{aligned}
\frac{\partial u}{\partial t}+\nabla \cdot(\vec{f}(u)) & =0, \\
u(0, \cdot) & =u_{0},
\end{aligned}
$$

where $u=u(t, \vec{x}): \mathbb{R}_{+}^{d+1}:=(0, \infty) \times \mathbb{R}^{d} \rightarrow P \subset \mathbb{R}^{m}$ is the desired solution $(P$ the set of physically reasonable values), $\vec{f}=\left(f^{i}\right), f^{i}: P \rightarrow \mathbb{R}^{m}$ is the (smooth) flux function, and $u_{0}: \mathbb{R}^{d} \rightarrow P$ is the initial data. Here and in the sequel " $\nabla, \Delta, . "$ are meant with respect to $\vec{x}$.

An important example of hyperbolic systems of conservation laws are the (nonisentropic) compressible Euler equations:

$$
\begin{aligned}
\rho_{t}+\nabla \cdot(\rho \vec{v}) & =0, \\
\left(\rho v^{i}\right)_{t}+\nabla \cdot\left(\rho v^{i} \vec{v}\right)+p_{x_{i}} & =0 \quad(i=1, \ldots, d), \\
(\rho e)_{t}+\nabla \cdot((\rho e+p) \vec{v}) & =0 .
\end{aligned}
$$

Here, $\rho$ is density, $\vec{v}=\left(v^{i}\right)$ is velocity, and $e$ is specific energy, which decomposes into

$$
e=\frac{|\vec{v}|^{2}}{2}+q
$$

Received by the editor November 7, 2004 and, in revised form, May 5, 2005.

2000 Mathematics Subject Classification. Primary 35L65, 35L67, 76L05, 76H05, 76N10.

Key words and phrases. Conservation law, well posedness, entropy solution, Riemann problem, shock, contact discontinuity, compressible Euler equations, entropy/entropy flux pair.

This material is based upon work supported by an SAP/Stanford Graduate Fellowship and by the National Science Foundation under Grant no. DMS 0104019. Any opinions, findings, and conclusions or recommendations expressed in this material are those of the author and do not necessarily reflect the views of the National Science Foundation.

(C)2006 American Mathematical Society Reverts to public domain 28 years from publication 
the first summand is specific kinetic energy, and $q$ is specific internal energy. The pressure is a function of $\rho, q$; a common choice is the polytropic pressure law

$$
p=(\gamma-1) \rho q
$$

$\left(1<\gamma \leq \frac{5}{3}\right.$; for air, $\left.\gamma=\frac{7}{5}\right)$. The set of admissible values is

$$
P=\{q>0, \rho>0\} .
$$

It is well known that (11) and (2) need not have a global smooth solution, even if the initial data $u_{0}$ is smooth. For this reason, one has to study weak solutions, defined as functions $u \in L_{\text {loc }}^{1}\left(\mathbb{R}_{+}^{d+1} ; P\right)$ that satisfy

$$
-\int_{0}^{\infty} \int_{\mathbb{R}^{d}} u \frac{\partial \phi}{\partial t}+\vec{f}(u) \cdot \nabla \phi d \vec{x} d t=\int_{\mathbb{R}^{d}} u_{0}(\vec{x}) \phi(0, \vec{x}) d \vec{x},
$$

for all test functions $\phi \in C_{c}^{\infty}\left(\overline{\mathbb{R}_{+}^{d+1}}\right)$. Moreover, there can be more than one weak solution, so it is necessary to impose an additional condition, called the entropy condition, to single out a unique weak solution (the entropy solution).

One definition of entropy solutions is the vanishing viscosity (VV) definition; it requires that $u$ is the limit of the sequence $\left(u^{\epsilon}\right)_{\epsilon>0}$ of solutions of

$$
\begin{aligned}
\frac{\partial u^{\epsilon}}{\partial t}+\nabla \cdot\left(\vec{f}\left(u^{\epsilon}\right)\right) & =\epsilon \Delta u^{\epsilon} \quad \text { in } \mathbb{R}_{+}^{d+1}, \\
u^{\epsilon}(0, \cdot) & =u_{0} \quad \text { on }\{0\} \times \mathbb{R}^{d} .
\end{aligned}
$$

The limit is taken in some suitable topology, usually as a boundedly almost everywhere limit. We call such a function $u$ a $V V$ solution.

Another definition uses entropy/entropy flux (EEF) pairs $(\eta, \vec{\psi})$, where $\eta: P \rightarrow \mathbb{R}$ is a smooth strictly convex function, called entropy, whereas $\vec{\psi}=\left(\psi^{1}, \ldots, \psi^{d}\right)^{\prime}$ with smooth $\psi^{i}: P \rightarrow \mathbb{R}$ is called an entropy flux; $\eta$ and $\vec{\psi}$ are required to satisfy

$$
\frac{\partial \psi^{i}}{\partial u^{\alpha}}=\sum_{\beta=1}^{m} \frac{\partial \eta}{\partial u^{\beta}} \frac{\partial f^{i \beta}}{\partial u^{\alpha}} \quad(i=1, \ldots, d, \alpha=1, \ldots, m) .
$$

By multiplying (7) from the left with $\eta^{\prime}\left(u^{\epsilon}\right)$ and using (9), one obtains

$$
\frac{\partial\left(\eta \circ u^{\epsilon}\right)}{\partial t}+\sum_{i=1}^{d} \frac{\partial\left(\psi^{i} \circ u^{\epsilon}\right)}{\partial x^{i}}=\epsilon \Delta\left(\eta \circ u^{\epsilon}\right)-\epsilon \sum_{i=1}^{d} \eta^{\prime \prime}\left(u^{\epsilon}\right) \frac{\partial u^{\epsilon}}{\partial x^{i}} \frac{u^{\epsilon}}{\partial x^{i}} \leq \epsilon \Delta\left(\eta \circ u^{\epsilon}\right)
$$

(here, we used that $\eta$ is convex). Upon multiplying the last equation with a nonnegative test function $\phi$ and integrating by parts, this yields

$$
-\int_{0}^{\infty} \int_{\mathbb{R}^{d}} \eta\left(u^{\epsilon}\right) \frac{\partial \phi}{\partial t}+\vec{\psi}\left(u^{\epsilon}\right) \cdot \nabla \phi d \vec{x} d t \leq \epsilon \int_{0}^{\infty} \int_{\mathbb{R}^{d}} \eta\left(u^{\epsilon}\right) \Delta \phi d \vec{x} d t+\int_{\mathbb{R}^{d}} \eta\left(u_{0}\right) d \vec{x} .
$$

If, as assumed above, $\left(u^{\epsilon}\right) \rightarrow u$ boundedly almost everywhere, then (11) implies

$$
-\int_{0}^{\infty} \int_{\mathbb{R}^{d}} \eta(u) \frac{\partial \phi}{\partial t}+\vec{\psi}(u) \cdot \nabla \phi d \vec{x} d t \leq \int_{\mathbb{R}^{d}} \eta\left(u_{0}\right) d \vec{x} .
$$

Functions $u$ that satisfy (12) for all EEF flux pairs are called EEF solutions (of (11)). As we have shown, VV solutions are necessarily EEF solutions.

In the literature, the term entropy solution is used to refer either to EEF or to VV solutions, often without explicit mention, because it has been assumed that the two definitions are equivalent for the Euler equations and many other physically 
relevant systems (see Ser99] p. 101, Daf00] p. 49, GR96] p. 32; see the discussion in Section 5 for verified special cases). However, for the purpose of this paper it is necessary to distinguish the two notions, as we will discuss a possible numerical counterexample to their equivalence.

The (gas-dynamic) specific entropy $s$ is defined as

$$
\begin{aligned}
& s=\log q+(1-\gamma) \log \rho, \\
& \eta:=-\rho s, \quad \psi^{i}:=-\rho s v^{i}
\end{aligned}
$$

and provides an EEF pair for the Euler equations.

A common simplification is to assume that $s$ is constant in space and time. This yields the isentropic Euler equations

$$
\begin{aligned}
\rho_{t}+\nabla \cdot(\rho \vec{v}) & =0 \\
\left(\rho v^{i}\right)_{t}+\nabla \cdot\left(\rho v^{i} \vec{v}\right)+p_{x_{i}} & =0 \quad(i=1, \ldots, d)
\end{aligned}
$$

with

$$
p(\rho)=\rho^{\gamma} .
$$

In this case, $P=\{\rho>0\}$. An EEF pair is provided by the specific energy $e$,

$$
e=\frac{|\vec{v}|^{2}}{2}+\frac{\rho^{\gamma-1}}{\gamma-1}
$$

with

$$
\eta:=\rho e, \quad \psi^{i}:=(\rho e+p) v^{i}
$$

It is cumbersome to verify the EEF condition (12) directly, not to mention the $\mathrm{VV}$ condition. There are easier criteria for piecewise smooth functions, which we define in the following customized way:

Definition 1. (1) A point $(t, \vec{x}) \in \mathbb{R}_{+}^{d+1}$ is called a point of smoothness if $u$ is $C^{\infty}$ in a small neighbourhood of $(t, \vec{x})$.

(2) A point $(t, \vec{x}) \in \mathbb{R}_{+}^{d+1}$ is called a point of piecewise smoothness of $u$ if there is a $C^{\infty}$ diffeomorphism $\Phi$ of a ball $V$ around 0 in $\mathbb{R}^{d+1}$ onto a neighbourhood $B$ of $(t, \vec{x})=\Phi(0)$ so that $u \circ \Phi$ is $C^{\infty}$ on $B_{-}$and on $B_{+}$ (where $B_{ \pm}:=\Phi\left(V_{ \pm}\right), V_{ \pm}:=\left\{y \in V: y_{1} \gtrless 0\right\}$ ); for later use, let $S$ be the surface $\Phi\left(V \cap\left(\{0\} \times \mathbb{R}^{d}\right)\right), n=\left(n^{t}, \vec{n}\right) \in \mathbb{R}^{d+1}$ a unit normal to $S$ in $(t, \vec{x})$ pointing into $B_{+}$; let $u_{+}, u_{-}$be the one-sided limits of $u$ in $(t, \vec{x})$ within $B_{-}$ resp. $\left.B_{+}\right)$. We also require $\vec{n} \neq 0$.

(3) $u$ is called piecewise smooth if there is a set $N$ of $d$-dimensional Hausdorff measure 0 so that all points in $\mathbb{R}_{+}^{d+1}-N$ are points of piecewise smoothness.

Proposition 1. Let $u$ be piecewise smooth. $u$ is an EEF solution of (1) if and only if

(1) it is a (classical) solution of (1) in each point of smoothness,

(2) $u(t, \cdot) \rightarrow u_{0}$ in $L_{\mathrm{loc}}^{1}$ as $t \downarrow 0$, and

(3) in each point $(t, x)$ of piecewise smoothness it satisfies the Rankine-Hugoniot conditions

$$
\left(u_{+}-u_{-}\right) n^{t}+\left(\vec{f}\left(u_{+}\right)-\vec{f}\left(u_{-}\right)\right) \cdot \vec{n}=0
$$

and (for all EEF pairs $(\eta, \vec{\psi})$ )

$$
\left(\eta\left(u_{+}\right)-\eta\left(u_{-}\right)\right) n^{t}+\left(\vec{\psi}\left(u_{+}\right)-\vec{\psi}\left(u_{-}\right)\right) \cdot \vec{n} \leq 0 .
$$


Proposition 1 is well known (see, for example, Section 11.1.1 in [Eva98]), as is the following property:

Proposition 2. For the Euler equations (3), resp. (15) (with polytropic gas law (51), resp. (16)), (18) is equivalent to the simpler condition that the normal velocity does not increase across discontinuities:

$$
\left(\vec{v}_{+}-\vec{v}_{-}\right) \cdot \vec{n} \leq 0 .
$$

The Cauchy problem for the Euler equations has several important symmetry properties, including the following:

Proposition 3. Let $u=\left(\rho, \vec{v}^{\prime}, q\right)^{\prime}$ be a weak solution for initial data $u_{0}=\left(\rho_{0}, \vec{v}_{0}^{\prime}, \vec{q}_{0}\right)^{\prime}$.

(1) Change of inertial frame: for all $\vec{w} \in \mathbb{R}^{d},\left(\rho(x+\vec{w} t),(\vec{v}(x+\vec{w} t, t)-\vec{w})^{\prime}\right.$, $q(x+\vec{w} t))^{\prime}$ is a weak solution for the same initial data $u_{0}$.

(2) Self-similarity: a function $f: \mathbb{R}_{+}^{d+1} \rightarrow \mathbb{R}^{m}$ is called self-similar if $f(r t, r \vec{x})$ $=f(t, \vec{x})$ for all $r>0$; the same for functions on $\mathbb{R}^{d}$. If the initial data is self-similar, then for any $r>0, u(r \vec{x}, r t)$ is a weak solution for the same initial data $u_{0}$.

These symmetries remain true after replacing "weak" by "VV" or "EEF". Analogous symmetries hold for the isentropic case.

\section{EXAMPLE AND NUMERICAL RESUltS}

Consider the following set $u_{0}$ of initial data for (3) with $d=2$ (see Figure 11): the data is symmetric under reflection across the $x$-axis and constant in each of four cones centered in the origin (in particular, constant along rays starting in the origin). In the origin, two shocks emanate into the first and fourth quadrant; the area on the left is supersonic inflow (parallel to the $x$-axis); the two areas on the

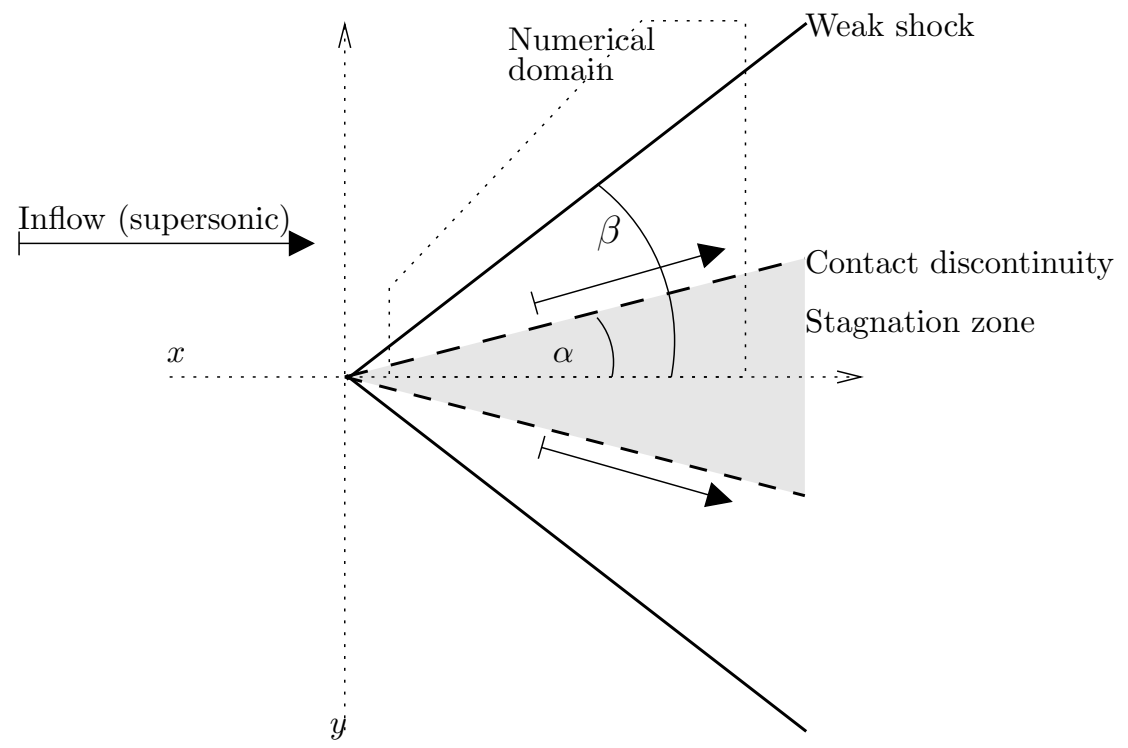

Figure 1. Solution T: this initial data is also a steady and selfsimilar solution for the compressible Euler equations in 2D. 
other side of the shocks are denser and hotter gas, moving parallel to the contact discontinuities (see CF48, Chapter IV C on choosing pre- and post-shock values that satisfy the Rankine-Hugoniot conditions; we choose the ones that yield the weaker shock). The gas in the stagnation area (enclosed by the contact discontinuities) has the same pressure as the post-shock gas on the other side, but with velocity $\vec{v}=0$. It is easy to check, using Propositions 1 and 2, that the steady solution $u(t, \vec{x})=u_{0}(\vec{x})$ is an EEF solution of (3)), resp. (15). Henceforth we refer to it as Solution $T$ (for theoretical).

However, instead of Solution T, numerical calculations produce the markedly different result in Figure 2 which we call Solution $N$ (for numerical) in the sequel (of course it is not known to be an exact solution). The numerical domain in Figure 2 is indicated as the dotted quadrilateral in Figure 1 . Figure 2 was computed as follows: adaptive refinement was used to achieve a better resolution at the same computational cost. To reduce numerical viscosity the grid was chosen so that near the right domain boundary the edges are aligned with the contact discontinuity and the shock. In order to capture self-similarity, the computations were done for a grid

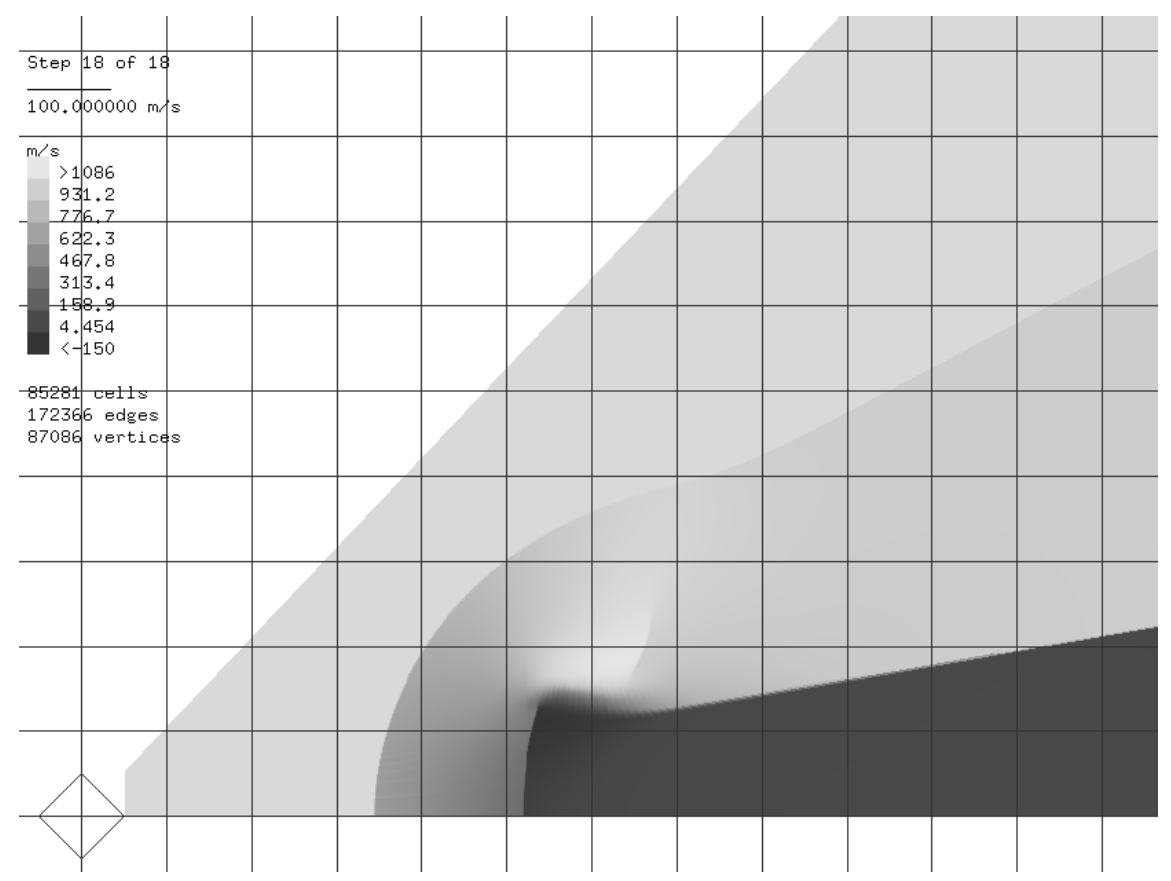

Figure 2. Solution N: (Rotate clockwise by $90^{\circ}$ to align with dotted area in Figure 1) Each square in the coordinate grid corresponds to a $100 \mathrm{~m} / \mathrm{s} \times 100 \mathrm{~m} / \mathrm{s}$ square in the $\vec{\xi}$ plane. The origin is marked by a diamond (lower left corner). Plotted: horizontal velocity. Godunov scheme for isentropic Euler equations; data: $\gamma=1.4, \alpha=10^{\circ}$; inflow: $\rho=1.19 \mathrm{~kg} / \mathrm{m}^{3}, v=1000 \mathrm{~m} / \mathrm{s}$, $T=20^{\circ} \mathrm{C}$. The solution differs significantly from Figure1, The results for nonisentropic Euler equations or other numerical schemes are similar. 
with moving vertices with coordinates $\vec{x}=t \vec{\xi}$ ( $\vec{\xi}$ has the dimension of a velocity; its components are called similarity coordinates). The moving-edge modifications discussed in [Ell00, Section 2.1.6] and [El105, Chapter 4] were used (the essential idea is to compute numerical fluxes across a moving edge by transforming to a steady edge, using invariance under change of inertial frame (Proposition 3), and to apply an arbitrary approximate Riemann solver to the transformed problem). The domain boundaries were chosen so that small perturbations on them propagate into the domain $(\vec{\xi} \cdot \vec{n}$ ( $\vec{n}$ outer unit normal) in each boundary point is larger than the maximum of $|\vec{v}|+c$ in the domain). This allows us to prescribe all components of the fluxes on the boundary.

Experiments with various modifications were made: changing the numerical scheme (the experiments were repeated for the Godunov scheme God59, the OsherSolomon scheme OS82, the ENO-RF scheme [SO89], and a second-order MUSCL code based on the first-order ENO-RF scheme), adding more numerical dissipation, refining uniformly rather than adaptively, using a Cartesian grid including origin and lower half-plane, or calculating in space rather than similarity coordinates. None of these modifications change the numerical results significantly; in all cases, the numerical results converge to the same Solution N.

Solution N appears to be self-similar (i.e., steady in similarity coordinates), but it is strongly unsteady, so it is clearly different from Solution T.

\section{Conclusions about numerical methods}

While the discrepancy between Solutions N and T opens many new problems, we can already draw one definite conclusion.

If we assume that Solution $\mathrm{T}$ is the correct solution, many ${ }^{1}$ popular numerical schemes fail to converge to physical solutions. Although it cannot be ruled out that they ultimately converge to Solution $\mathrm{T}$ as the numerical grid becomes infinitely fine, they approach Solution $\mathrm{N}$ for computationally accessible grids - which is all that matters for practical purposes.

On the other hand, if we assume that Solution $\mathrm{N}$ is the correct solution, there is a trivial theoretical example of misconvergence: consider the (semidiscrete) Godunov scheme on grids whose edges are exactly aligned with the discontinuities of Solution $\mathrm{T}$ (see Figure 1): in exact arithmetic it would have Solution $\mathrm{T}$ as a steady state on every grid.

In either case - even if Solution $\mathrm{N}$ is correct, which would be less catastrophic for numerical analysis - we have to conclude that discrete entropy inequalities are not sufficient to avoid convergence to unphysical solutions on feasible grids. Hence they lose a bit of their value as design principles for numerical schemes, although they are still useful as easy-to-check necessary conditions that are sufficient for scalar conservation laws and (probably) 1D systems (as supported by the recent work on small total variation solutions described in Section (5).

Although many reports of deficiencies of various numerical schemes have been published, the clear case of failure observed here has no precedent.

\footnotetext{
${ }^{1}$ In the sense of every scheme that was tested.
} 


\section{TheORETICAL INTERPRETATION}

It remains to discuss which of Solution $\mathrm{T}$ and Solution $\mathrm{N}$ is the physical one and, if Solution T is correct, what causes numerical schemes to produce Solution N. There are three possible explanations (which are not mutually exclusive):

(1) either Solution $\mathrm{N}$ is an example of failure of numerical methods, or

(2) EEF solutions are not stable (in the sense of continuous dependence on initial data), or

(3) EEF solutions are not unique.

4.1. Breakdown of numerical methods. It has already been shown in Section 3 that the Godunov scheme is flawed, in the sense that it can fail to converge to the physical solution on feasible grids. Hence it is natural to suspect that Solution $\mathrm{N}$ is a numerical artifact that does not correspond to a seccond EEF solution (or any other type of solution of the Euler equations).

Solution T is steady and self-similar. "Steadyness" is a nongeneric property that is usually not inherited by finite-accuracy numerical solutions (for example for a Riemann problem that is solved exactly by a single shock, most numerical schemes produce small additional waves and a slightly different shock). However, one would expect numerical approximations to be at least almost steady, unlike Solution N.

In a single space dimension, the conservation property of numerical schemes often guarantees accurate shock locations, even if the overall accuracy of the scheme is poor. On the other hand, in two or more dimensions numerical imprecision can significantly change the shape and location of shocks. This may be the cause of Solution N. For example, the upwards deflection of the incoming flow by the high-pressure area in front of the stagnation region could be weaker in numerical calculations than in Solution T; the additional pressure would cause the stagnation region to collapse.

However, in this case the numerical results would depend strongly on the choice of numerical method, mesh width, and other parameters. This is not observed; rather, all choices produce essentially the same results.

4.2. Instability. A second explanation is instability (in the sense of lack of continuous dependence on the initial data). It is possible that Solution $\mathrm{N}$ is an approximation to an unsteady EEF Solution $T^{\prime}$ that results from a slight perturbation of Solution $\mathrm{T}$ at initial time (such perturbations are inevitable in most numerical computations due to inexact arithmetic, discretization error, artificial viscosity, etc.) Since Solution N is produced (up to minor differences) for any "perturbation" (i.e., for any choice of mesh, numerical method and parameters), it would indicate that Solution $\mathrm{T}$ constitutes a set of initial values for which the Euler equations are not stable.

On inspection in similarity coordinates, it appears that the numerical solutions are bounded, converge quickly to Solution N, and remain steady (many orders of magnitude of time have been observed), so Solution $T^{\prime}$ would have to be at least approximately self-similar. If we assume it is asymptotically self-similar for large time, then the following theorem implies that the asymptote is an EEF solution:

Theorem 1. Let $u \in L^{\infty}\left(\mathbb{R}_{+}^{d+1}\right)$ be an EEF solution of (6). Assume that $u$ is asymptotically self-similar (see Definition 2); then its asymptotic limit $w$ (a selfsimilar function) is an EEF solution as well. 
(The proof of Theorem 1 and an analogous result for steady solutions are presented in the Appendix.) The asymptote would assume the same initial data as Solution T, but would have to be different from it (by closeness to Solution N). This would already imply the third explanation (nonuniqueness of the EEF solution).

To avoid this, it is necessary to assume either that Solution $\mathrm{T}^{\prime}$ is approximately, but not asymptotically self-similar-for example it might oscillate periodically around some self-similar function without approaching it - or that the selfsimilar asymptote has data at infinity that does not match the initial data (Solution T). Either of these cases would be revealed by a sufficiently fine numerical grid: the numerical computation on that grid would refuse to converge to a steady state for the given boundary data. But the mesh used to compute Solution $\mathrm{N}$ is already rather fine, as can be seen from the curved shocks in Figure 2, there is no obvious reason why an even better grid is required.

4.3. Nonuniqueness of EEF solutions. The third explanation is nonuniqueness: Solution N corresponds to an EEF solution that assumes the same initial data as Solution T.

In this context, the following peculiarity is important: according to Proposition 1. the EEF condition (12) is "insensitive" to sets with $(d-1)$-dimensional Hausdorff measure 0 (such as a single point, for $d=2$ ), e.g., if (12) is satisfied for

$$
\phi \in C_{c}^{\infty}\left(\overline{\mathbb{R}_{+}^{d+1}}-\{0\}\right),
$$

it is satisfied for all

$$
\phi \in C_{c}^{\infty}\left(\overline{\mathbb{R}_{+}^{d+1}}\right) .
$$

In verifying that Solution $\mathrm{T}$ is an EEF solution, we may ignore the rather singular wedge tip in the origin of Figure 1. It is counterintuitive that conditions for the physical correctness of solutions may ignore such singularities.

It seems unlikely that the solution in Figure 1 is stable under small perturbations at the origin (such as perturbations from viscous terms in the VV limit). Note that a planar shock, with inflow state on one side and stagnation area state on the other side, would not be steady but move into the stagnation zone quickly - it seems unlikely that the example data, which has less mass and energy and more $x$-momentum in the $\{x>0\}$ halfplane, would yield a steady pattern in the origin (however, the "maximum principle" implicit in this argument is merely heuristic and may be wrong in some instances).

But it is the following observation that provides the strongest argument for nonuniqueness: the Lax-Wendroff theorem (see LW60; see also GR96, KRW96 and most generally [Ell03 for Lax-Wendroff-type theorems for irregular grids) states that if a numerical scheme is consistent and satisfies a discrete entropy inequality (see HHL76, MO79, OC84, Tad84, Tad87, OT88), then the limit of a boundedly almost everywhere converging sequence of numerical solutions is an EEF solution. (Note that we prescribe the full numerical flux on the boundaries, so the boundary conditions are analogous to an initial condition and can be treated with a straightforward modification of the Lax-Wendroff theorem for initial-value problems.) The Godunov scheme, used to compute Figure 2 is the standard example for a consistent scheme that satisfies all discrete entropy inequalities. Our numerical solutions do, on inspection, appear to converge quickly; this would imply that Solution N corresponds to an EEF solution. 


\section{Related WORK}

For multi-dimensional scalar $(m=1)$ conservation laws with arbitrary $f$, Kru70 (generalizing earlier work) shows that a global EEF solution exists, is unique, satisfies the $\mathrm{VV}$ condition as well, and is stable under $L^{1}$ perturbations of the initial data.

Gli65 provides a famous existence proof for strictly hyperbolic systems with genuinely nonlinear fields and initial data with small total variation; the interaction functionals constructed in this paper are a crucial ingredient for all subsequent work. Liu81 extends the result to systems with linearly and some nonlinearly degenerate fields. BCP00] constructed the Standard Riemann Semigroup (SRS), an $L^{1}$-stable semigroup of EEF solutions for initial data with small total variation, for strictly hyperbolic systems with genuinely nonlinear or linearly degenerate fields (see also [LY99]). [BL97] showed that EEF solutions to 1D systems are unique and coincide with the SRS solutions, under certain smoothness assumptions including small total variation (see also BG99]). BB01 proves that for small $T V$ initial data and strictly hyperbolic (but otherwise arbitrary) systems VV solutions exist and are stable under $L^{1}$ perturbations of the initial data, so for the class of solutions that are subject both to [BL97] and to BB01, EEF and VV solutions are equivalent.

On the other hand, an EEF pair $(\eta, \vec{\psi})$ has to satisfy the condition (9) which is an overdetermined problem for $m \geq 3$, so for some systems no EEF pairs exist and the EEF condition is void. However, EEF pairs do exist for most physically relevant systems, even those with $m \geq 3$. More seriously, for certain $2 \times 2$ systems (with nonlinear degenerate fields) CL81] constructs a single weak shock that is an EEF solution but does not satisfy the Liu entropy condition (see Liu74, Liu75]). By BB01, there must be a VV solution (for the same initial data) that satisfies the Liu entropy condition as well - so it cannot be the aforementioned weak shock. Therefore the example in [CL81] also constitutes an example of a nonunique EEF solution, albeit for an "artificial" system with nonlinear degeneracy.

Hop67 proposes the EEF condition for scalar conservation laws $(m=1)$, proves that it is implied by the $\mathrm{VV}$ condition under some circumstances, and notes that there is a large set of convex entropies. Apparently independently, Kru70 obtained analogous results for systems. Lax71 contains the first use of the term "entropy condition" for the EEF condition. Various forms of the EEF condition had been known and in use for special systems such as the Euler equations for a long time (e.g., by the name of Clausius-Duhem inequality), especially as shock relations; however, the above references seem to be the first to define the general notion of strictly convex EEF pairs, to propose the EEF condition as a mathematical tool for arbitrary systems of conservation laws and to formulate it in the weak form (12) rather than the special case (18).

LZY98 provides an analytical and numerical discussion of 2D Riemann problems for various systems including the Euler equations. However, they focus on data constant in each of the four quadrants, so Solution $\mathrm{T}$ is not covered.

\section{Conjectures AND Final REMARKS}

The results demonstrate that

the Godunov method does not always converge to the physical solution on feasible grids. 
Moreover, they suggest the following conjecture:

EEF solutions to the multi-dimensional Euler equations are not always unique.

If this conjecture is true, it would have far-reaching consequences. The EEF condition would not be sufficient as a selection principle for physical/unique solutions, except in special cases like the ones described in Section 5. It would be necessary to find ways to use the cumbersome VV condition or to discover new entropy conditions.

Although the numerical results support the conjecture unambiguously, the question is so important that a rigorous proof is highly desirable. However, since the initial data has large vorticity at the contact discontinuity, it seems difficult to construct (or to prove results about) exact solutions. One possible line of attack is to derive novel entropy conditions from the VV condition and to check whether they are violated by the steady solution in Figure 1.

In any case, this paper motivates the investigation of multi-dimensional Riemann problems for systems; these appear to be very difficult and exhibit a large variety of phenomena (see [LL98, LZY98]). This goal requires techniques for proving the existence of smooth steady or self-similar solutions to boundary-value problems for systems of nonlinear hyperbolic conservation laws; while there are classical methods for smooth solutions in hyperbolic regions, work on tools for the elliptic and mixed case has begun only recently (see, e.g., [EL05]).

\section{Appendix: Asymptotically STEAdy AND SELF-Similar WEAK SOLUTIONS}

Remark. In Theorem 1 and in the following statements,

$$
f(t, \cdot) \rightarrow g \quad \text { in } L_{\mathrm{loc}}^{1}(\Omega)
$$

as $t \downarrow 0$ resp. $t \uparrow \infty$ is to be understood as: for all $\epsilon>0$ and $K \Subset \Omega$ there is a $T=T(\epsilon)>0$ so that for almost all $0<t \leq T$, resp. $t \geq T$,

$$
\|f(t, \cdot)-g\|_{L^{1}(K)} \leq \epsilon .
$$

Lemma 1. Let $u \in L^{\infty}\left(\mathbb{R}_{+}^{d+1}\right), u_{0} \in L^{\infty}\left(\mathbb{R}^{d}\right)$. If

(1) $u(t, \cdot) \rightarrow u_{0}$ in $L_{\mathrm{loc}}^{1}\left(\mathbb{R}^{d}\right)$ as $t \downarrow 0$, and

(2) u satisfies (6) for all $\phi \in C_{c}^{\infty}\left(\underline{0}, \infty\left[\times \mathbb{R}^{d}\right)\right.$,

then $u$ satisfies (6) for all $\phi \in C_{c}^{\infty}\left(\left[0, \infty\left[\times \mathbb{R}^{d}\right)\right.\right.$.

Proof. Let $\theta \in C^{\infty}[0, \infty[$ so that $\theta=1$ on $[0,1]$ and $\theta=0$ on $[2, \infty[$. For any $T>0$, define $\theta_{T}(t):=\theta\left(T^{-1} t\right)$. Note that $\left|\theta_{T}\right|=O(1),\left|\theta_{T}^{\prime}\right|=O\left(T^{-1}\right)$ (as $T \downarrow 0$ ). For given $\phi \in C_{c}^{\infty}\left(\left[0, \infty\left[\times \mathbb{R}^{d}\right)\right.\right.$, split $\phi_{1}(t, x):=\theta_{T}(t) \phi(t, x)$ and $\phi_{2}=\phi-\phi_{1}$.

$$
\begin{aligned}
\int_{0}^{\infty} & \int_{\mathbb{R}^{d}} u \phi_{t}+\vec{f}(u) \cdot \nabla \phi d x d t \\
= & \int_{0}^{2 T} \int_{\mathbb{R}^{d}} u \phi_{1 t}+\vec{f}(u) \cdot \nabla \phi_{1} d x d t \\
& +\int_{T}^{\infty} \int_{\mathbb{R}^{d}} u \phi_{2 t}+\vec{f}(u) \cdot \nabla \phi_{2} d x d t .
\end{aligned}
$$


Since supp $\phi_{2} \subset\left[T, \infty\left[\times \mathbb{R}^{d}\right.\right.$, the second summand vanishes by assumption. The first summand equals

$$
\begin{aligned}
& =O\left(T \sup _{0<t \leq 2 T}\left\|u(t, \cdot)-u_{0}\right\|_{1} \cdot\left(T^{-1}+1\right)\right)+\int_{0}^{2 T} \int_{\mathbb{R}^{d}} u_{0}(x) \phi_{1 t}(t, x) d x d t \\
& =O\left(\sup _{0<t \leq 2 T}\left\|u(t, \cdot)-u_{0}\right\|_{1}\right)-\int_{\mathbb{R}^{d}} u_{0}(x) \phi(0, x) d x .
\end{aligned}
$$

On taking $T \downarrow 0$, all $O$ terms vanish; hence $u$ satisfies (6).

Definition 2. (1) A function $u \in L_{\text {loc }}^{1}\left(\mathbb{R}_{+}^{d+1} ; \mathbb{R}^{m}\right)$ is called asymptotically selfsimilar if there is a function $w: \mathbb{R}^{d} \rightarrow \mathbb{R}^{m}$ so that

$$
u\left(t, t^{-1} \cdot\right) \rightarrow w \quad \text { in } L_{\mathrm{loc}}^{1}\left(R^{d}\right) \text { as } t \uparrow \infty .
$$

(2) $u$ is called self-similar if, for some $w, u\left(t, t^{-1} \cdot\right)=w$ for almost all $t>0$.

Proof of Theorem 1. To show that $w$ is a weak solution it is sufficient to check that

$$
\int_{0}^{\infty} \int_{\mathbb{R}^{d}} w\left(\frac{x}{t}\right) \phi_{t}(t, x)+\vec{f}\left(w\left(\frac{x}{t}\right)\right) \cdot \nabla \phi(t, x) d x d t=0
$$

for all $\left.\phi \in C_{c}^{\infty}\right] 0, \infty[$. The essential idea is to scale coordinates to shift the support of $\phi$ into a large- $t$ region and to use asymptotic convergence.

Let $0<t_{1}<t_{2}$ be such that $\operatorname{supp} \phi \subset\left[t_{1}, t_{2}\right] \times \mathbb{R}^{d}$. Let $\epsilon>0$ be arbitrary, and set $T=T(\epsilon)$ (see above). The change of coordinates $t=\frac{t_{1}}{T} \tau, x=\frac{t_{1}}{T} \xi$ changes the left-hand side of (19) into

$$
\begin{aligned}
\left(\frac{t_{1}}{T}\right)^{d+1} & \int_{T}^{\frac{t_{2} T}{t_{1}}} \int_{\mathbb{R}^{d}} w\left(\frac{\xi}{\tau}\right) \phi_{t}\left(\frac{t_{1}}{T} \tau, \frac{t_{1}}{T} \xi\right) \\
& +\vec{f}\left(w\left(\frac{\xi}{\tau}\right)\right) \cdot \nabla \phi\left(\frac{t_{1}}{T} \tau, \frac{t_{1}}{T} \xi\right) d \xi d \tau \\
= & \left(\frac{t_{1}}{T}\right)^{d+1} \int_{T}^{\frac{t_{2} T}{t_{1}}} \int_{\mathbb{R}^{d}} u(\tau, \xi) \phi_{t}\left(\frac{t_{1}}{T} \tau, \frac{t_{1}}{T} \xi\right) \\
& +\vec{f}(u(\tau, \xi)) \cdot \nabla \phi\left(\frac{t_{1}}{T} \tau, \frac{t_{1}}{T} \xi\right) d \xi d \tau \\
& +O\left(\left(\frac{t_{1}}{T}\right)^{d+1} \cdot T \cdot \epsilon T^{d}\right),
\end{aligned}
$$

where $O$ is with respect to $\epsilon \downarrow 0$. Note that the support of the scaled $\phi$ is in $\left[T, \infty\left[\times \mathbb{R}^{d}\right.\right.$. Also, the assumption that $u$ is bounded is essential here. The first summand on the right-hand side equals (up to a factor)

$$
\int_{T}^{\frac{t_{2} T}{t_{1}}} \int_{\mathbb{R}^{d}} u(\tau, \xi) \frac{T}{t_{1}} \phi\left(\frac{t_{1}}{T} \tau, \frac{t_{1}}{T} \xi\right)_{\tau}+\vec{f}(u(\tau, \xi)) \cdot \nabla_{\xi}\left(\phi\left(\frac{t_{1}}{T} \tau, \frac{t_{1}}{T} \xi\right)\right) d \xi d \tau .
$$

Since $u$ is assumed to be a weak solution, this term vanishes. Taking $\epsilon \downarrow 0$ in (20) yields (19).

For the proof of the EEF part, replace $u, w$ by $\eta(u), \eta(w)$ and $f(u), f(w)$ by $\psi(u), \psi(w)$ above.

An analogous theorem can be obtained for steady rather than self-similar solutions. 
Definition 3. (1) $u \in L_{\text {loc }}^{1}\left(\mathbb{R}_{+}^{d+1} ; \mathbb{R}^{m}\right)$ is called steady if, for some $w: \mathbb{R}^{d} \rightarrow$ $\mathbb{R}^{m}, u(t, \cdot)=w$ for almost all $t>0$.

(2) $u \in L_{\text {loc }}^{1}\left(\mathbb{R}_{+}^{d+1} ; \mathbb{R}^{m}\right)$ is called asymptotically steady if there is a $w: \mathbb{R}^{d} \rightarrow$ $\mathbb{R}^{m}$, so that

for almost all $t \geq T$.

$$
u(t, \cdot) \rightarrow w \quad \text { in } L_{\mathrm{loc}}^{1}\left(\mathbb{R}^{d}\right)
$$

Theorem 2. If $u \in L^{\infty}\left(\mathbb{R}_{+}^{d+1} ; \mathbb{R}^{m}\right)$ is an asymptotically steady and bounded weak solution, then $w$ (as in Definition 3) is a weak solution as well. If $u$ is an EEF solution, so is $w$.

Proof. Let $\phi \in C_{c}^{\infty}\left((0, \infty) \times \mathbb{R}^{d}\right)$ be arbitrary. Let $\operatorname{supp} \phi \subset[0, \tau]$. For any $\epsilon>0$,

$$
\begin{aligned}
\int_{0}^{\infty} & \int_{\mathbb{R}^{d}} w(x) \phi_{t}(t, x)+\vec{f}(w(x)) \cdot \nabla \phi(t, x) d x d t \\
= & \int_{0}^{\infty} \int_{\mathbb{R}^{d}} u(T+t, x) \phi_{t}(t, x) \\
& +\vec{f}(u(T+t, x)) \cdot \nabla \phi(t, x) d x d t+O\left(\tau \epsilon\|D \phi\|_{\infty}\right) \\
= & O\left(\tau \epsilon\|D \phi\|_{\infty}\right),
\end{aligned}
$$

because we can extend $\phi(\cdot-T, \cdot) \in C_{c}\left((T, \infty) \times \mathbb{R}^{d}\right)$ smoothly by 0 to a map $\tilde{\phi} \in C_{c}\left((0, \infty) \times \mathbb{R}^{d}\right)$ and use the fact that $u$ is a weak solution. Lemma 1 shows that $w$ is a weak solution.

For the proof of the EEF part, replace $u, w$ by $\eta(u), \eta(w)$ and $f(u), f(w)$ by $\psi(u), \psi(w)$ above.

\section{ACKNOWLEDGMENTS}

The author would like to thank Tai-Ping Liu for his support and comments and Ron Fedkiw, Doron Levy, Wolfgang Dahmen and Ralf Massjung for sharing their insight about numerical schemes.

\section{REFERENCES}

[BB01] S. Bianchini and A. Bressan, Vanishing viscosity solutions of nonlinear hyperbolic systems, Tech. report, S.I.S.S.A., Trieste, Italy, 2001.

[BCP00] A. Bressan, G. Crasta, and B. Piccoli, Well-posedness of the Cauchy problem for $n \times n$ systems of conservation laws, Memoirs AMS, no. 694, American Mathematical Society, July 2000 .

[BG99] A. Bressan and P. Goatin, Oleinik type estimates and uniqueness for $n \times n$ conservation laws, J. Diff. Eqs. 156 (1999), 26-49.

[BL97] A. Bressan and P. LeFloch, Uniqueness of weak solutions to systems of conservation laws, Arch. Rat. Mech. Anal. 140 (1997), 301-317.

[CF48] R. Courant and K.O. Friedrichs, Supersonic flow and shock waves, Interscience Publishers, 1948.

[CL81] J.G. Conlon and Tai-Ping Liu, Admissibility criteria for hyperbolic conservation laws, Indiana Univ. Math. J. 30 (1981), no. 5, 641-652.

[Daf00] C. Dafermos, Hyperbolic conservation laws in continuum physics, Springer, 2000.

[EL05] V. Elling and Tai-Ping Liu, The ellipticity principle for selfsimilar potential flow, J. Hyper. Diff. Eqns. (2005), to appear, Preprint arxiv:math.AP-0509332.

[El100] V. Elling, Numerical simulation of gas flow in moving domains, Diploma Thesis, RWTH Aachen (Germany), 2000.

[Ell03] — A Lax-Wendroff type theorem for unstructured quasiuniform grids, 2003, arxiv:math.NA/0509331. 
[Ell05] _ A Lax-Wendroff type theorem for unstructured grids, Ph.D. Dissertation, Stanford University, 2005.

[Eva98] L.C. Evans, Partial differential equations, American Mathematical Society, 1998.

[Gli65] J. Glimm, Solutions in the large for nonlinear hyperbolic systems of equations, Comm. Pure Appl. Math. 18 (1965), 697-715.

[God59] S. K. Godunov, A finite difference method for the numerical computation of discontinuous solutions of the equations of fluid dynamics, Mat. Sb. 47 (1959), 271-290.

[GR96] E. Godlewski and P.-A. Raviart, Numerical approximation of hyperbolic systems of conservation laws, Springer, 1996.

[HHL76] A. Harten, J.M. Hyman, and P.D. Lax, On finite-difference approximation and entropy conditions for shocks, Comm. Pure Appl. Math. 29 (1976), 297-321.

[Hop67] E. Hopf, On the right weak solution of the Cauchy problem for quasi-linear equations of first order, J. Math. Mech. 17 (1967), 483-487.

[Kru70] S.N. Kružkov, First order quasilinear equations in several independent variables, Mat. Sb. 81 (1970), no. 2, 285-355, transl. in Math. USSR Sb. 10 (1970) no. 2, 217-243.

[KRW96] D. Kröner, M. Rokyta, and M. Wierse, A Lax-Wendroff type theorem for upwind finite volume schemes in 2-D, East-West J. Numer. Math. 4 (1996), 279-292.

[Lax71] P.D. Lax, Shock waves and entropy, Contributions to Nonlinear Functional Analysis (E.A. Zarantonello, ed.), Academic Press, 1971, pp. 603-634.

[Liu74] Tai-Ping Liu, The Riemann problem for general $2 \times 2$ conservation laws, Trans. Amer. Math. Soc. 199 (1974), 89-112.

[Liu75] _ The Riemann problem for general systems of conservation laws, J. Diff. Eqs. 18 (1975), 218-234.

[Liu81] - Admissible solutions of hyperbolic conservation laws, Memoirs AMS, no. 240, American Mathematical Society, 1981.

[LL98] P.D. Lax and Xu-Dong Liu, Solution of two-dimensional Riemann problems of gas dynamics by positive schemes, SIAM J. Sci. Comput. 19 (1998), no. 2, 319-340.

[LW60] P. Lax and B. Wendroff, Systems of conservation laws, Comm. Pure Appl. Math. 13 (1960), 217-237.

[LY99] Tai-Ping Liu and Tong Yang, Well-posedness theory for hyperbolic conservation laws, Comm. Pure Appl. Math. 52 (1999), 1553-1586.

[LZY98] Jiequan Li, Tong Zhang, and Shuli Yang, The two-dimensional Riemann problem in gas dynamics, Addison Wesley Longman, 1998.

[MO79] A. Majda and S. Osher, Numerical viscosity and the entropy condition, Comm. Pure Appl. Math. 32 (1979), 797-838.

[OC84] S. Osher and S. Chakravarthy, High resolution schemes and the entropy condition, SIAM J. Numer. Anal. 21 (1984), no. 5, 955-984.

[OS82] S. Osher and F. Solomon, Upwind difference schemes for hyperbolic systems of conservation laws, Math. Comp. 38 (1982), 339-373.

[OT88] S. Osher and E. Tadmor, On the convergence of difference approximations to scalar conservation laws, Math. Comp. 50 (1988), no. 181, 19-51.

[Ser99] D. Serre, Systems of conservation laws, vol. 1, Cambridge University Press, 1999.

[SO89] C. W. Shu and S. Osher, Efficient implementation of essentially non-oscillatory shockcapturing schemes, II, J. Comp. Phys. 83 (1989), 32-78.

[Tad84] E. Tadmor, Numerical viscosity and the entropy condition for conservative difference schemes, Math. Comp. 43 (1984), no. 168, 369-381.

[Tad87] The numerical viscosity of entropy stable schemes for systems of conservation laws, I, Math. Comp. 49 (1987), no. 179, 91-103.

Division of Applied Mathematics, Brown University, 182 George Street, Providence, Rhode IsLand 02912

E-mail address: velling@stanfordalumni.org 